\title{
Advanced Nanomaterials for Applications in Photonic and Sensor Devices
}

\author{
Sheng-Joue Young $\mathbb{D}^{1}$ Shoou-Jinn Chang $\mathbb{D},{ }^{2}$ and Yi-Hsing Liu ${ }^{2}$ \\ ${ }^{1}$ Department of Electronic Engineering, National United University, Miaoli City 36063, Taiwan \\ ${ }^{2}$ Institute of Microelectronics, Department of Electrical Engineering, National Cheng Kung University, Tainan 701, Taiwan
}

Correspondence should be addressed to Sheng-Joue Young; shengjoueyoung@gmail.com

and Shoou-Jinn Chang; changsj@mail.ncku.edu.tw

Received 21 January 2022; Accepted 21 January 2022; Published 12 February 2022

Copyright (C) 2022 Sheng-Joue Young et al. This is an open access article distributed under the Creative Commons Attribution License, which permits unrestricted use, distribution, and reproduction in any medium, provided the original work is properly cited.

In recent years, nanostructures for photonic/sensor device applications have been increasingly developed because of their flexibility and light weight for daily use, making them deployable. Therefore, photonic/sensor devices have been the subject of much interest. Photonic/sensor devices are environmentally sustainable, considering the availability of nanostructured raw materials. The main goal of this special issue is to discover new scientific knowledge relevant to IOT-based intelligent sensor systems and applied materials in nanoscience and nanotechnology.

This special issue collected five excellent papers. The published papers are introduced as follows.

Prof. C.-T. Yen et al. reported on "Development of a Continuous Blood Pressure Measurement and Cardiovascular Multi-Indicator Platform for Asian Populations by Using a Back Propagation Neural Network and Dual Photoplethysmography Sensor Signal Acquisition Technology." This study proposed a measurement platform for continuous blood pressure estimation based on dual photoplethysmography sensors and a back propagation neural network (BPNN) that can be used for the continuous and rapid measurement of blood pressure and analysis of cardiovascularrelated indicators. To increase the robustness of the BPNN model, the authors input data of 100 Asian participants into the training database, including those with and without cardiovascular disease, each with a proportion of approximately $50 \%$. The proposed real-time blood pressure measurement system exhibited a mean accuracy of $98.22 \%$ and $95.58 \%$ for SBP and DBP, respectively.
Prof. K.-T. Lam et al. reported on "Characteristics of Metal-Semiconductor-Metal Ultraviolet Photodetectors Based on Pure ZnO/Amorphous IGZO Thin-Film Structures." In this study, metal-semiconductor-metalstructured ultraviolet (UV) photodetectors (PDs) based on pure zinc oxide $(\mathrm{ZnO})$ and amorphous indium gallium $\mathrm{ZnO}$ (a-IGZO) thin films were fabricated and characterized. The $\mathrm{ZnO}$ seed layers were deposited on corning glass substrates via a radio frequency magnetron sputtering technique. Results showed that under a $5 \mathrm{~V}$ applied bias, the dark currents of the pure $\mathrm{ZnO}$ and a-IGZO thin films were $0.112 \mathrm{pA}$ and $2.85 \mathrm{nA}$, respectively. Meanwhile, the UV-tovisible rejection ratios of the pure $\mathrm{ZnO}$ and a-IGZO thin films were 14.33 and 256, respectively. Finally, the PDs of thea-IGZO thin films had a lower leakage current and higher rejection ratio than the pure $\mathrm{ZnO}$ thin films from the UV-tovisible light region.

Q. M. Thi Tran et al. reported on "Biosynthesis of Zinc Oxide Nanoparticles Using Aqueous Piper betle Leaf Extract and Its Application in Surgical Sutures." In this study, the authors aimed to synthesize $\mathrm{ZnO}$ nanoparticles (NPs) by using Piper betle leaf extract and used it to coat the suture. The effect of synthesis parameters on the size and morphology of the $\mathrm{ZnO}$ NPs was also analyzed. The UV-Vis spectrum indicated the formation of $\mathrm{ZnO} \mathrm{NPs}$ at approximately $370 \mathrm{~nm}$. The volume of leaf extract plays a role in controlling the size and morphology of ZnO NPs. The average particle size of the as-synthesized $\mathrm{ZnO}$ NPs was approximately $112 \mathrm{~nm}$ with a hexagonal and spherical shape. The results proved that 
the ZnO NPs performed a high antibacterial activity against Staphylococcus aureus and Escherichia coli with its antibacterial effectiveness up to 5 days. The $\mathrm{ZnO}$ NP-coated sutures also exhibited a high performance on bacterial inactivation. With key findings, this study largely contributed to lowering the burden on medical services in terms of medical treatment cost in developing countries.

A. Ortiz-Dosal et al. reported on "Determination of the Immunoglobulin G Spectrum by Surface-Enhanced Raman Spectroscopy Using Quasispherical Gold Nanoparticles.” This study is aimed at determining the Raman spectrum of IgG at physiological concentrations using quasispherical gold NPs as a surface-enhanced Raman spectroscopy (SERS) substrate. Methods. We initially determined the Raman spectrum of IVIG at 5\%. Subsequently, for SERS characterization, decreasing dilutions of the protein were prepared by adding deionized water and an equal volume of the $5 \mathrm{~nm}$ gold quasispherical NP colloid. For each protein concentration, the Raman spectrum was determined using a $10 \mathrm{x}$ objective. We focused the 532 and $785 \mathrm{~nm}$ laser on the sample surface in a range of $500-1800 \mathrm{~cm}^{-1}$, with five acquisitions and an acquisition time of $30 \mathrm{~s}$. Results. We obtained the IVIG spectrum using SERS up to a concentration of $75 \mathrm{mg} / \mathrm{dL}$. The Raman bands correspond to aromatic amino acid side chains and the characteristic beta-sheet structure of IgG. Conclusion. The use of $5 \mathrm{~nm}$ quasispherical gold NPs as a SERS substrate allows for detecting the Raman spectrum of IVIG at physiological concentrations.

N. M. Noah reported on "Design and Synthesis of Nanostructured Materials for Sensor Applications." The development of sensor devices with improved characteristics, such as sensitivity, low cost, fast response, reliability, rapider recovery, reduced size, in situ analysis, and simple operation, has attracted increasing attention. Nanostructured materials have shown great potential in improving these properties for chemical and biological sensors. Different nanostructured materials have been used in manufacturing nanosensors, which include nanoscale wires (capability of high detection sensitivity), carbon nanotubes (very high surface area and high electron conductivity), thin films, metal and metal oxide NPs, polymer, and biomaterials. This review provides different methods that have been used in the synthesis and fabrication of these nanostructured materials followed by an extensive review of the recent developments of metal, metal oxides, carbon nanotubes, and polymer nanostructured materials in sensor applications.

\section{Conflicts of Interest}

We, the guest editorial team of the special issue, declare that we do not have any conflict of interest or private agreements with companies.

\section{Acknowledgments}

The guest editors would like to thank the authors for their contributions to this special issue and all the reviewers for their constructive comments.
Sheng-Joue Young Shoou-Jinn Chang

Yi-Hsing Liu 